\title{
Prenatal management, pregnancy and pediatric outcomes in fetuses with septated cystic hygroma
}

\author{
C.Y. Sanhal ${ }^{1,2}$, I. Mendilcioglu ${ }^{1,2}$, M. Ozekinci ${ }^{1}$, S. Yakut ${ }^{3}$, Z. Merdun ${ }^{1}$, M. Simsek $^{1,2}$ and G. Luleci ${ }^{3}$ \\ ${ }^{1}$ Department of Obstetrics and Gynecology, Faculty of Medicine, Akdeniz University, Antalya, Turkey \\ ${ }^{2}$ Department of Perinatology, Faculty of Medicine, Akdeniz University, Antalya, Turkey \\ ${ }^{3}$ Department of Medical Biology and Genetics, Faculty of Medicine, Akdeniz University, Antalya, Turkey
}

\begin{abstract}
It has been reported that, compared with simple increased nuchal translucency, fetal cases with septated cystic hygroma $(\mathrm{CH})$ are more likely to face perinatal handicaps. However, pediatric outcomes and proper prenatal counseling for this anomaly have not yet been truly defined. We performed this study to determine pregnancy and pediatric outcomes of fetuses with septated $\mathrm{CH}$. We searched records for cases with septated $\mathrm{CH}$ and collected data for structural abnormalities, karyotype analysis, and pregnancy outcomes. Fetuses born with septated $\mathrm{CH}$ were also evaluated for their pediatric outcomes. Sixty-nine fetuses with septated $\mathrm{CH}$ were enrolled in the study. Results showed that chromosomal abnormalities were present in 28 fetuses (40.6\%), and the most common aneuploidy was Turner syndrome $(n=14,20.3 \%) ; 16(23.2 \%)$ of the remaining cases, in which aneuploidy was not found, had coexistent structural malformations; $25(36.2 \%)$ cases had normal karyotype and morphology. The total number of live births and infants with unfavorable neurologic follow-up were $13(18.8 \%)$ and 2 (2.9\%), respectively. Septated $\mathrm{CH}$ is associated with poor perinatal outcomes; therefore, karyotype analysis and ultrasonographic anomaly screening should be performed as initial steps, and expectant management should be offered to couples with euploid fetuses that have normal morphology.
\end{abstract}

Key words: Septated; Cystic; Hygroma; Perinatal; Outcomes; Neurologic

\section{Introduction}

Cystic hygroma $(\mathrm{CH})$ or hygroma colli is characterized by abnormal accumulation of fluid in the region of the fetal neck and is one of the major anomalies associated with aneuploidy $(1,2)$. Extensive usage of ultrasound screening has increased the rate of detection of $\mathrm{CH}$ in pregnancies (3).

Prenatal diagnosis of $\mathrm{CH}$ via ultrasound is based on demonstration of a bilateral, mostly symmetric, cystic structure located in the occipitocervical region, with the lesion either septated by internal trabeculae (septated) or not (nonseptated) (4). It was formerly asserted that obstruction in the fetal cervical lymphatics may trigger the accumulation of lymphatic fluid and the degree of obstruction may lead to the presence and extensiveness of septations. Incomplete obstruction with temporary blockage, which can resolve spontaneously with high pressure, may cause a nonseptated $\mathrm{CH}$, whereas complete obstruction may account for a septated $\mathrm{CH}$ $(5,6)$. On the other hand, some authors claimed that septations could not be used to distinguish between increased nuchal translucency and $\mathrm{CH}$, and thus they could be seen within the translucency in all fetuses (7). Even so, "septated CH"' is a commonly used term, and data regarding this anomaly are expanding.

Ultrasonographic diagnosis of septated $\mathrm{CH}$ is relatively easy, and chromosomal analysis - either with chorion villus sampling (CVS) or amniocentesis - is generally accepted as the sequential step in its management. Unfortunately, recommendations after these procedures are not yet thoroughly defined, especially in the euploid group. We carried out this retrospective study in order to define the pregnancy and pediatric outcomes in fetuses and infants with septated $\mathrm{CH}$.

\section{Material and Methods}

This retrospective study was conducted based on a review of medical records of patients who had fetal

Correspondence: C.Y. Sanhal, Department of Obstetrics and Gynecology, Faculty of Medicine, Akdeniz University, Dumlupinar Street, Konyaalti, 07059 Antalya, Turkey. Fax: +90-242-227-4482. E-mail: cemsanhal@yahoo.com 
septated $\mathrm{CH}$, diagnosed by ultrasonography in the Department of Obstetrics and Gynecology, Akdeniz University, Turkey, between March 2000 and December 2013. All scans were performed by two experienced maternal-fetal medicine experts using a Voluson 730 Expert TM (GE Healthcare, USA) multifrequency convex transducer at 2.0-7.0 MHz.

The former description by Malone et al. (8) - an enlarged hypoechoic space at the back of the fetal neck, extending along the length of the fetal back, in which septations are clearly visible - was used as the definition of septated $\mathrm{CH}$ (Figure 1). Within the diagnosis, all patients were informed of an association between $\mathrm{CH}$ and fetal aneuploidy and were offered fetal karyotyping. A complete fetal sonographic examination was then performed for documentation of other structural anomalies. CVS and amniocentesis procedures were performed in patients between 11-14 and 16-20 weeks of gestation, respectively. Karyotypes of fetuses and pregnancy and pediatric outcome data were collected either from the records at the Obstetrics and Gynecology, Genetics, and Pediatrics Departments, at Akdeniz University, Turkey, or from phone calls.

\section{Results}

Within a 13-year period, a total of 72 pregnant women carrying fetuses with fetal septated $\mathrm{CH}$ were detected. All patients underwent cytogenetic analysis. CVS was performed for the pregnant women at 11-14 weeks of gestation $(n=53)$, while amniocentesis was achieved for the cases at $16-20$ weeks $(n=19)$. Three patients were excluded due to inadequate pregnancy and pediatric outcome data.

Table 1 shows chromosomal abnormalities of fetuses with septated $\mathrm{CH}$. Twenty-eight of 69 cases (40.6\%) were aneuploid. Turner syndrome was the most common

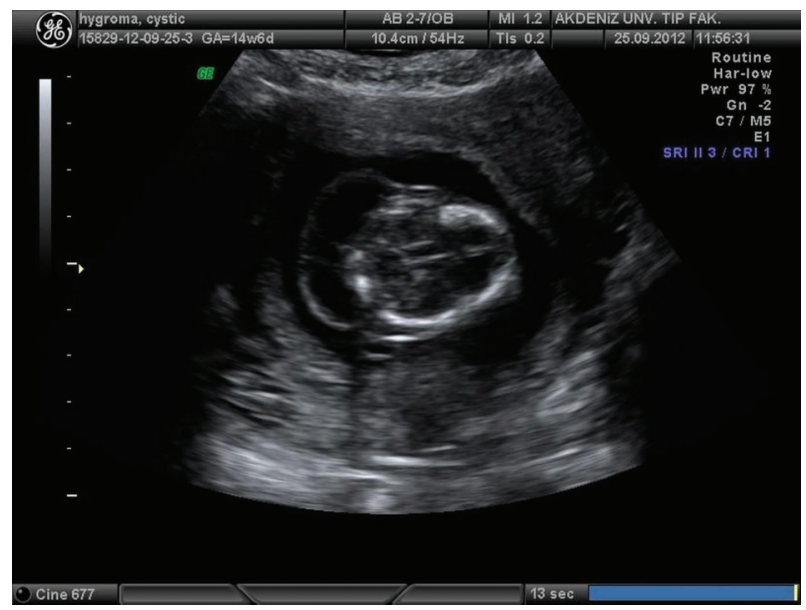

Figure 1. Ultrasonographic view of fetal septated cystic hygroma. karyotype abnormality detected, but trisomy 21 was also diagnosed frequently. Table 1 also exhibits the structural malformations diagnosed among the remaining 41 cases in which aneuploidy was not found. Ascites, pleural effusion, or hydrops were the leading major structural fetal malformations. Altogether, the rate of either chromosomal or major structural abnormalities in fetuses with septated $\mathrm{CH}$ was $63.8 \%$.

Table 2 presents pregnancy and pediatric outcomes of fetuses with septated $\mathrm{CH}$. A total of 44 of 69 (63.8\%) pregnancies were terminated electively. Moreover, spontaneous fetal demise occurred during the follow-up of 11 patients $(15.9 \%)$.

The total number of live births was 13 (13/69; 18.8\%). There was no chromosomal abnormality in this group, except one fetus with mosaicism of Turner syndrome $(45, X[15] / 46, X X[35])$, and this child was doing well at the age of 4 years. Additionally, structural malformations had been diagnosed prenatally in two other cases. The first case had hypertrophic cardiomyopathy, tricuspid-mitral regurgitation, and the second had tetralogy of Fallot. They were 4 and 2 years old, respectively, and both had normal neurologic follow-up. Furthermore, eight infants had completely normal pediatric outcomes. The median follow-up duration of these cases was 36 months (range 2-84 months). Two infants had developmental delay and motor retardation without any certain etiology. One of these two cases was 6 months old with prenatally detected bilateral hydronephrosis, and the other case was 2 years old without any coexistent abnormalities.

Table 3 demonstrates the outcomes of fetuses with septated $\mathrm{CH}$ and normal karyotype and morphology. Only 10 of 25 cases (40\%) were born alive in this group, and there was only one infant $(4 \%)$ with an unfavorable neurologic follow-up.

\section{Discussion}

$\mathrm{CH}$, which was first described in 1828 by Redenbacher (9), is an anomaly of the lymphatic system that is characterized by single or multiple cysts within the soft tissue, ranging in size from several millimeters to $80 \mathrm{~mm}$ and containing a clear or cloudy fluid akin to lymph. It occupies a prominent place in the category of chromosomal abnormality-related congenital malformations $(10,11)$. Today, there is no accurate consensus either for the classification of $\mathrm{CH}$ or for the management of pregnancies in which the fetus has this lesion. The main debate about the nomenclature of subtypes arises from the existence of sonographically detected septations and also prognostic differences between septated and nonseptated $\mathrm{CH}$. Although some authors have declared that septations are not convenient for the differentiation of increased nuchal translucency and $\mathrm{CH}$ due to common occurrence in both conditions, others support the use of the terms "septated $\mathrm{CH}$ " and "nonseptated $\mathrm{CH}^{\text {" or }}$ 
Table 1. Septated cystic hygroma: chromosomal and structural abnormalities $(n=69)$.

\begin{tabular}{lrr}
\hline Outcome & Number & $\%$ \\
\hline Chromosomal abnormality & $28 / 69$ & 40.6 \\
Turner syndrome & $14 / 28$ & 50.0 \\
Trisomy 21 & $9 / 28$ & 32.1 \\
Trisomy 18 & $3 / 28$ & 10.7 \\
Trisomy 13 & $1 / 28$ & 3.6 \\
Other (mosaic Turner) & $1 / 28$ & 3.6 \\
Structural malformation & $16 / 69$ & 23.2 \\
Ascites, pleural effusion or hydrops & $5 / 16$ & 31.3 \\
Hydrocephalus & $1 / 16$ & 6.3 \\
Arthrogyrposis & $1 / 16$ & 6.3 \\
Corpus callosum agenesis & $1 / 16$ & 6.3 \\
Pes equinovarus & $1 / 16$ & 6.3 \\
Diaphragmatic hernia & $1 / 16$ & 6.3 \\
Amniotic band syndrome & $1 / 16$ & 6.3 \\
Mesomelia & $1 / 16$ & 6.3 \\
Hypertrophic cardiomyopathy, tricuspid-mitral regurgitation & $1 / 16$ & 6.3 \\
Hypoplastic left heart & $1 / 16$ & 6.3 \\
Fallot's tetralogy & $1 / 16$ & 6.3 \\
Bilateral hydronephosis & $1 / 16$ & 6.3 \\
\hline
\end{tabular}

"increased nuchal translucency" because of the differences in the comparative outcomes of aneuploidy (5 times more likely), cardiac malformations (12 times more likely), and fetal or neonatal death (6 times more likely) of the pregnancies complicated by these anomalies $(7,8)$. We agree with this latter viewpoint and we designed this study by discriminating and excluding fetuses with nonseptated $\mathrm{CH}$.

Our findings showed that the frequency of aneuploidy accompanying septated $\mathrm{CH}$ was $40.6 \%$. Previous studies, which used the same definition of septated $\mathrm{CH}$, reported similar results. Graesslin et al. (12) and Gedikbasi et al. (13) reported $52.7 \%$ and $47.6 \%$ for the abnormal karyotype rate of this group, respectively. Fifty percent of our aneuploid cases corresponded to Turner syndrome, $32.1 \%$ to Down syndrome, and $10.7 \%$ to trisomy 18 . This distribution was also in accordance with the series in which Turner syndrome was the most common form among aneuploid cases of septated $\mathrm{CH}(14,15)$.

In addition to aneuploidy, coexistence of septated $\mathrm{CH}$

Table 2. Septated cystic hygroma: pregnancy and pediatric outcome $(n=69)$.

\begin{tabular}{lcc}
\hline Outcome & Number & $\%$ \\
\hline Elective pregnancy termination & $44 / 69$ & 63.8 \\
Chromosomal abnormality & $25 / 44$ & 56.9 \\
Structural malformation & $11 / 44$ & 25 \\
Normal karyotype with normal morphology & $8 / 44$ (4 co-twin) & 18.2 \\
Spontaneous intrauterine fetal demise & $11 / 69$ & 15.9 \\
Chromosomal abnormality & $2 / 11$ & 18.2 \\
Structural malformation & $2 / 11$ & 18.2 \\
Spontaneous demise fetus with normal morphology & $7 / 11$ & 63.6 \\
Live births & $13 / 69$ & 18.8 \\
Chromosomal abnormality (mosaic Turner) & $1 / 13$ & 7.7 \\
Cardiac abnormality & $2 / 13$ & 15.4 \\
Developmental delay/motor retardation & $2 / 13$ & 15.4 \\
Normal postnatal neurologic outcome & $11 / 69$ & 15.9 \\
Ongoing pregnancy (normal morphology, normal karyotype) & $1 / 69$ & 1.4 \\
\hline
\end{tabular}


Table 3. Outcome of fetuses with septated cystic hygroma and normal karyotype morphology $(n=25)$.

\begin{tabular}{lcr}
\hline Outcome & Number & $\%$ \\
\hline Elective pregnancy termination & $8 / 25$ & 32 \\
Spontaneous intrauterine fetal demise & $7 / 25$ & 28 \\
Normal postnatal neurologic outcome & $8 / 25$ & 32 \\
Developmental delay/motor retardation & $1 / 25$ & 4 \\
Ongoing pregnancy & $1 / 25$ & 4 \\
\hline
\end{tabular}

and structural malformations, especially cardiac abnormalities, was also described $(15,16)$. Besides fetal ascites, pleural effusion, or hydrops, cardiac abnormalities were the second most common group of structural malformations in our study. These findings support the need for fetal echocardiogram and anomaly screening in a case of septated $\mathrm{CH}$.

Today in routine practice among the vast majority of maternal-fetal medicine specialists, karyotype analysis and anomaly screening are accepted as the initial steps in the evaluation of a fetus with $\mathrm{CH}$. Management choices are limited, and it is relatively easy to offer termination of pregnancy to the parents with an aneuploid or a structurally malformed fetus with septated $\mathrm{CH}$. In our study, 28 fetuses suffered from chromosomal abnormality. Two of these fetuses died spontaneously, and the parents of all remaining fetuses, except one with mosaicism of Turner syndrome, accepted pregnancy termination. There were also 16 structurally malformed fetuses, five of which were aneuploid and terminated. Only three fetuses with bilateral hydronephrosis, tetralogy of Fallot, and hypertrophic cardiomyopathy with tricuspid mitral regurgitation were born alive. The infants with cardiac abnormalities had normal postnatal neurologic outcomes, whereas severe mental and motor retardation developed at the hydronephrotic infant's follow-up without any definite reason. Other euploid malformed cases died spontaneously or were terminated.

Furthermore, there were $25(36.2 \%)$ fetuses with normal karyotype and normal morphology in our study. Of these, although their fetus did not have any karyotype or structural abnormalities, eight couples chose pregnancy termination, after our disclosure about the possible postnatal progress based on results from other studies, including the coexistence of $\mathrm{CH}$ and cardiac abnormality, neurologic impairment, and late-onset postnatal disorders, even if those unintended consequences were not common $(17,18)$. Seven fetuses also aborted spontaneously.

Finally, a total of $11(15.9 \%)$ infants had normal postnatal neurologic adequacy. Nine of them were euploid and structurally nonmalformed cases, i.e., among the euploid and structurally nonmalformed group, if they could survive until birth, 9 of 10 (90\%) fetuses with septated $\mathrm{CH}$ had completely normal postnatal neurologic follow-up. However, we should indicate that this result could be affected by the outcomes of those fetuses whose parents chose pregnancy termination instead of continuing gestation. Nevertheless, this satisfactory finding was in accordance with the results, which were reported in the study conducted by Malone et al. (8), with the largest fetal septated $\mathrm{CH}$ series in this field.

We found a number of articles revealing the recurrence of first trimester septated $\mathrm{CH}$. One report described three consecutive pregnancies complicated by $\mathrm{CH}$ with nonimmune hydrops and normal karyotype (19). Recurrent cases were also depicted as associated with Fryns syndrome (diaphragmatic hernia and digital hypoplasia) (20). Moreover, recurrent $\mathrm{CH}$ was reported to have a possible autosomal recessive pattern (21). However, none of our subjects had $\mathrm{CH}$ recurrence.

Chromosomal microarray analysis is a relatively new method that measures the alterations in DNA throughout the human genome. It has been considered as a first-tier test for examining children with developmental and intellectual disabilities, congenital anomalies, and autism spectrum (22). Compared to ordinary karyotyping, comparative genomic hybridization (CGH), a type of microarray used in prenatal testing in some selected laboratories, reveals additional clinically significant abnormalities in approximately $6 \%$ of fetuses with ultrasonographic abnormalities and a normal conventional karyotype $(23,24)$. Therefore, $\mathrm{CGH}$ analysis can be recommended to the patients with a fetus with one or more major structural abnormality identified by ultrasonographic examination and to the women with intrauterine fetal demise or stillbirth when further cytogenetic analysis is desired (25). Unfortunately, none of the patients in our study had CGH analysis.

Despite published data, the counseling of couples with fetal septated $\mathrm{CH}$ after normal karyotype analysis and anomaly screening is quite challenging. This situation gave rise to limitations of our study when elective pregnancy terminations were chosen, particularly in euploid and nonmalformed groups. Additionally, early cessation of gestation might affect the rate of detection of intrauterine anomalies.

In conclusion, we want to emphasize that the outcome of a pregnancy complicated by fetal septated $\mathrm{CH}$ is poor, but couples can be encouraged to complete the pregnancy with some future risks regarding neurologic development of the infant, especially when the fetus is euploid and structurally normal. Further studies are needed to accurately distinguish between outcomes for fetuses, which might be adequate or unfavorable, and to provide more substantial information to the couples. 


\section{References}

1. Chen CP, Chien SC. Prenatal sonographic features of Turner syndrome. J Med Ultrasound 2007; 15: 251-257, doi: 10.1016/S0929-6441(08)60042-9.

2. De Vigan C, Baena N, Cariati E, Clementi M, Stoll C. Contribution of ultrasonographic examination to the prenatal detection of chromosomal abnormalities in 19 centres across Europe. Ann Genet 2001; 44: 209-217, doi: 10. 1016/S0003-3995(01)01091-7.

3. Snijders RJ, Noble P, Sebire N, Souka A, Nicolaides KH. UK multicentre project on assessment of risk of trisomy 21 by maternal age and fetal nuchal-translucency thickness at 10-14 weeks of gestation. Fetal Medicine Foundation First Trimester Screening Group. Lancet 1998; 352: 343-346, doi: 10.1016/S0140-6736(97)11280-6.

4. Bianca S, Bartoloni G, Boemi G, Barrano B, Barone C, Cataliotti $A$, et al. Familial nuchal cystic hygroma without fetal effects: Genetic counselling and further evidence for an autosomal recessive subtype. Congenit Anom 2010; 50: 139-140, doi: 10.1111/j.1741-4520.2010.00273.x.

5. Brumfield CG, Wenstrom KD, Davis RO, Owen J, Cosper P. Second-trimester cystic hygroma: prognosis of septated and nonseptated lesions. Obstet Gynecol 1996; 88: 979982, doi: 10.1016/S0029-7844(96)00358-4.

6. Bernstein HS, Filly RA, Goldberg JD, Golbus MS. Prognosis of fetuses with a cystic hygroma. Prenat Diagn 1991; 11: 349-355, doi: 10.1002/pd.1970110603.

7. Molina FS, Avgidou K, Kagan KO, Poggi S, Nicolaides KH. Cystic hygromas, nuchal edema, and nuchal translucency at 11-14 weeks of gestation. Obstet Gynecol 2006; 107: 678-683, doi: 10.1097/01.AOG.0000201979.23031.32.

8. Malone FD, Ball RH, Nyberg DA, Comstock CH, Saade GR, Berkowitz RL, et al. First-trimester septated cystic hygroma: prevalence, natural history, and pediatric outcome. Obstet Gynecol 2005; 106: 288-294, doi: 10.1097/01.AOG. 0000173318.54978.1f.

9. Masood SN, Masood MF. Case report of fetal axillo-thoracoabdominal cystic hygroma. Arch Gynecol Obstet 2010; 281: 111-115, doi: 10.1007/s00404-009-1068-2.

10. D'Alton M, Cleary-Goldman J. First and second trimester evaluation of risk for fetal aneuploidy: the secondary outcomes of the FASTER Trial. Semin Perinatol 2005; 29: 240-246, doi: 10.1053/j.semperi.2005.06.006.

11. Shimada S, Yamada H, Hoshi N, Kobashi G, Okuyama K, Hanatani K, et al. Specific ultrasound findings associated with fetal chromosome abnormalities. Congenit Anom 2009; 49: 61-65, doi: 10.1111/j.1741-4520.2009.00224.x.

12. Graesslin O, Derniaux E, Alanio E, Gaillard D, Vitry F, Quereux C, et al. Characteristics and outcome of fetal cystic hygroma diagnosed in the first trimester. Acta Obstet Gynecol Scand 2007; 86: 1442-1446, doi: 10.1080/ 00016340701644843.

13. Gedikbasi A, Oztarhan K, Aslan G, Demirali O, Akyol A, Sargin A, et al. Multidisciplinary approach in cystic hygroma: prenatal diagnosis, outcome, and postnatal follow up. Pediatr Int 2009; 51: 670-677, doi: 10.1111/j.1442-200X. 2009.02846.x.
14. Ville $Y$, Lalondrelle C, Doumerc S, Daffos F, Frydman R, Oury JF, et al. First-trimester diagnosis of nuchal anomalies: significance and fetal outcome. Ultrasound Obstet Gynecol 1992; 2: 314-316, doi: 10.1046/j.1469-0705.1992. 02050314.x.

15. Bronshtein M, Zimmer EZ, Blazer S. A characteristic cluster of fetal sonographic markers that are predictive of fetal Turner syndrome in early pregnancy. Am J Obstet Gynecol 2003; 188: 1016-1020, doi: 10.1067/mob.2003.230.

16. Surerus E, Huggon IC, Allan LD. Turner's syndrome in fetal life. Ultrasound Obstet Gynecol 2003; 22: 264-267, doi: 10.1002/uog.151.

17. Tanriverdi HA, Ertan AK, Hendrik HJ, Remberger K, Schmidt W. Outcome of cystic hygroma in fetuses with normal karyotypes depends on associated findings. Eur $\mathrm{J}$ Obstet Gynecol Reprod Biol 2005; 118: 40-46, doi: 10.1016/ j.ejogrb.2004.04.005.

18. Shulman LP, Emerson DS, Grevengood C, Felker RE, Gross SJ, Phillips OP, et al. Clinical course and outcome of fetuses with isolated cystic nuchal lesions and normal karyotypes detected in the first trimester. Am J Obstet Gynecol 1994; 171: 1278-1281, doi: 10.1016/0002-9378 (94)90147-3.

19. Teague KE, Eggleston MK, Muffley PE, Gherman RB. Recurrent fetal cystic hygroma with normal chromosomes: case report and review of the literature. J Matern Fetal Med 2000; 9: 366-369, doi: 10.1002/1520-6661(200011/ 12) $9: 6<366:: A I D-M F M 1010>3.3 . C O ; 2-5$.

20. Hosli IM, Tercanli S, Rehder H, Holzgreve W. Cystic hygroma as an early first-trimester ultrasound marker for recurrent Fryns' syndrome. Ultrasound Obstet Gynecol 1997; 10: 422-424, doi: 10.1046/j.1469-0705.1997. 10060422.x.

21. Dallapiccola B, Zelante L, Perla G, Villani G. Prenatal diagnosis of recurrence of cystic hygroma with normal chromosomes. Prenat Diagn 1984; 4: 383-386, doi: 10.1002/pd.1970040510.

22. Miller DT, Adam MP, Aradhya S, Biesecker LG, Brothman AR, Carter NP, et al. Consensus statement: chromosomal microarray is a first-tier clinical diagnostic test for individuals with developmental disabilities or congenital anomalies. Am $J$ Hum Genet 2010; 86: 749-764, doi: 10.1016/j.ajhg. 2010.04.006.

23. Shaffer LG, Rosenfeld JA, Dabell MP, Coppinger J, Bandholz AM, Ellison JW, et al. Detection rates of clinically significant genomic alterations by microarray analysis for specific anomalies detected by ultrasound. Prenat Diagn 2012; 32: 986-995, doi: 10.1002/pd.3943.

24. Wapner RJ, Martin CL, Levy B, Ballif BC, Eng CM, Zachary $\mathrm{JM}$, et al. Chromosomal microarray versus karyotyping for prenatal diagnosis. N Engl J Med 2012; 367: 2175-2184, doi: 10.1056/NEJMoa1203382.

25. Committee Opinion No. 581: the use of chromosomal microarray analysis in prenatal diagnosis. Obstet Gynecol 2013; 122: 1374-1377, doi: 10.1097/01.AOG. 0000438962.16108.d1. 